Department of

Paediatrics, Surgery and Internal

Medicine, National

Taiwan University,

Taipei, Taiwan

$\mathrm{M}-\mathrm{H}$ Wu

$\mathrm{J}-\mathrm{L}$ Lin

J-K Wang

I-S Chiu

M-L Young

Correspondence to: of Paediatrics, National Taiwan University Hospital, No 7 Chung-Shan South Road, Taipei, Taiwan ROC 100 .

Accepted for publication 18 May 1995
Dr M-H Wu, Department

\section{Electrophysiological properties of dual atrioventricular nodes in patients with right atrial isomerism}

\author{
Mei-Hwan Wu, Jiunn-Lee Lin, Jou-Kou Wang, Ing-Sh Chiu, Ming-Lon Young
}

\begin{abstract}
There are reports that in right atrial isomerism the conduction system has paired sinus nodes and paired atrioventricular nodes. Electrophysiological studies were performed in two patients with right atrial isomerism. One patient had a delta wave on the surface electro-
\end{abstract}

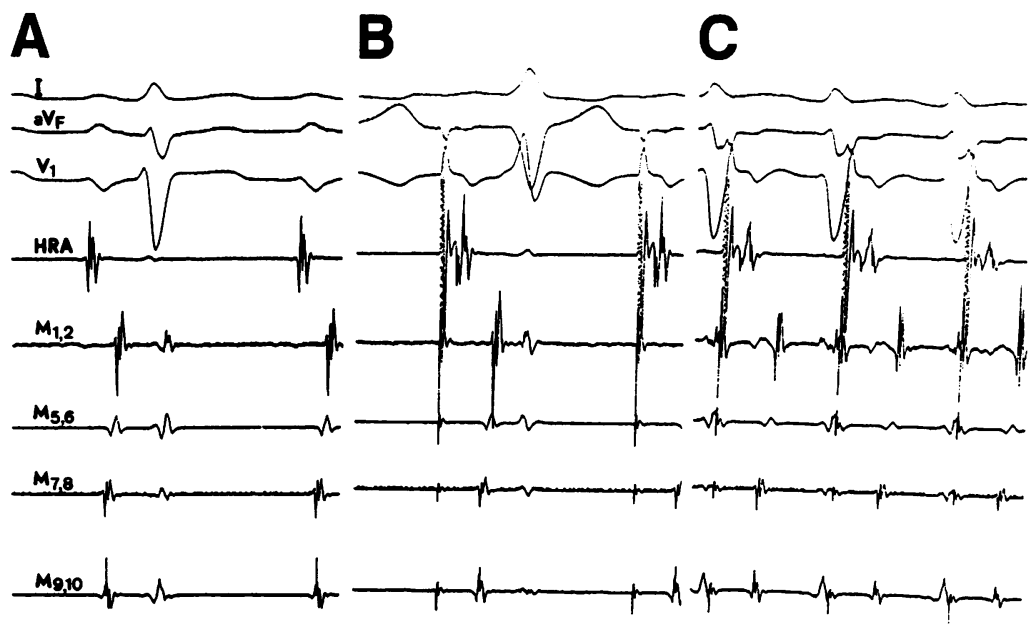

$\underline{R V}$

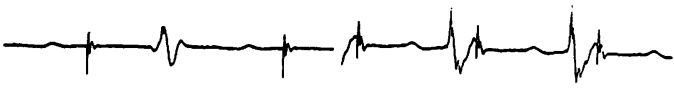

(1)

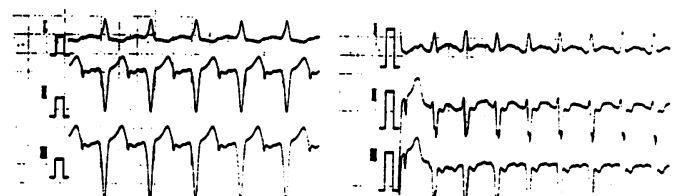
'Ifrmpimminim

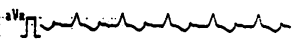

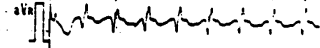

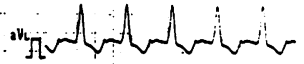

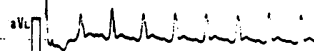

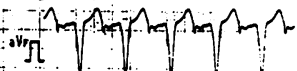

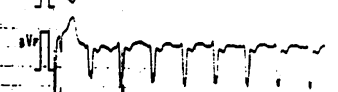

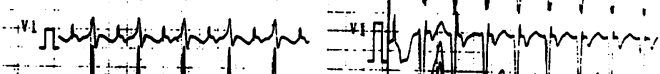
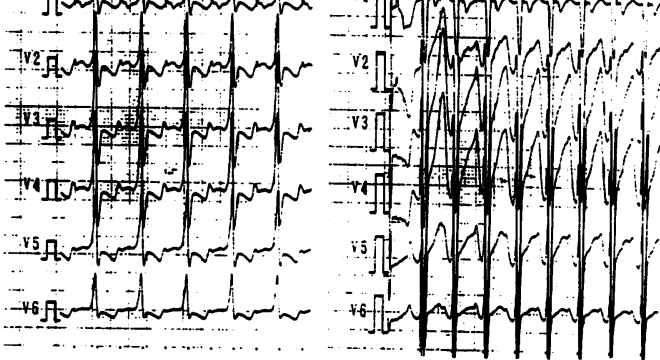

Figure 1 Electrocardiograms recorded at $(A)$ rest: atrioventricular conduction through the posterior atrioventricular node with a narrow $O R S$ complex and $r S$ pattern in V1. Electrocardiograms recorded during right atrial pacing at $520 \mathrm{~ms}(B):$ pre-excitation was evident from the short PR interval and delta wave. The polarity of delta wave and $Q R S$ complex suggested a left posterior accessory pathway. Electrocardiograms recorded during right atrial pacing at $340 \mathrm{~ms}$ (C) showing atrioventricular conduction through an anterior node with a narrow $Q R S$ complex and a $Q S$ pattern in V1. cardiogram without tachycardia attacks. The other, who did not have manifest pre-excitation, had recurrent narrow QRS tachycardia. Electrophysiological studies suggested the presence of dual atrioventricular nodes. Only unidirectional atrioventricular or ventriculoatrial conduction was demonstrated for these dual atrioventricular nodes even after infusion of isoprenaline. It is suggested that unidirectional conduction may be a common property of the dual atrioventricular nodes in right atrial isomerism and that the absence of retrograde ventriculoatrial conduction protects the patients against tachycardia.

(Br Heart f 1995;74:553-555)

Keywords: right atrial isomerism; dual atrioventricular nodes; electrophysiology.

Right atrial isomerism is usually associated with complex cyanotic congenital heart disease. ${ }^{1}$ The conduction system had been reported to have paired sinus nodes and dual atrioventricular (AV) nodes. ${ }^{23}$ However, the electrophysiological properties, to the best of our knowledge, have not been reported. We report two patients with asplenia, right atrial isomerism, total anomalous pulmonary venous return, complete atrioventricular canal, double outlet right ventricle, and pulmonary stenosis. One had a delta wave on the surface electrocardiogram without tachycardia attacks. The other, who did not have manifest preexcitation, had recurrent narrow QRS tachycardia. Electrophysiological studies suggested the presence of dual AV nodes.

\section{Case reports}

CASE 1

In an 8 year old boy who had been cyanosed since early infancy echocardiography and cardiac catheterisation showed right atrial isomerism, total anomalous pulmonary venous return draining back to right superior vena cava without obstruction, complete atrioventricular canal, double outlet right ventricle, and pulmonary stenosis. Additionally, a delta wave with a short PR interval was found on one electrocardiogram taken during follow up. None the less, he had never had tachycardia attacks.

Electrophysiological study was performed before the total cavopulmonary anastomosis 
A

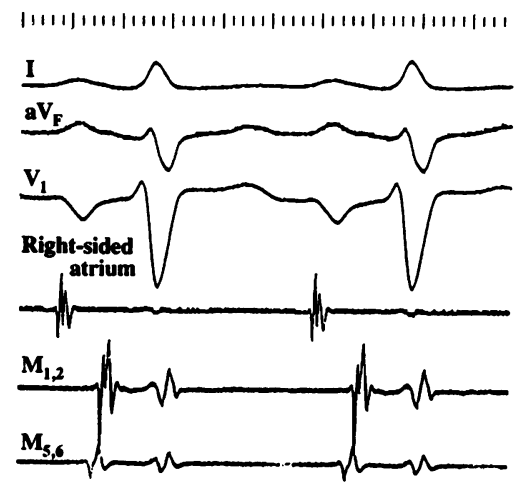

$\underline{M_{1, s}}$

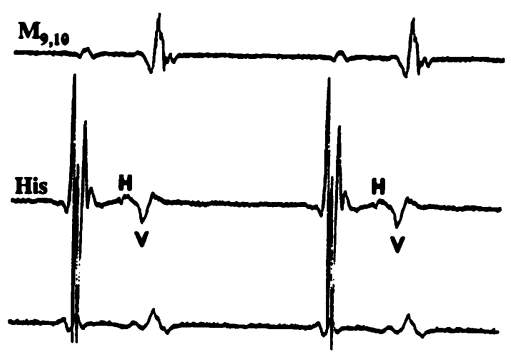

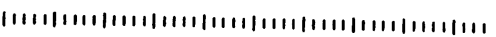

Figure 2 His potential recorded near the posterior atrioventricular node in case $1(A)$ and case 2 (B).

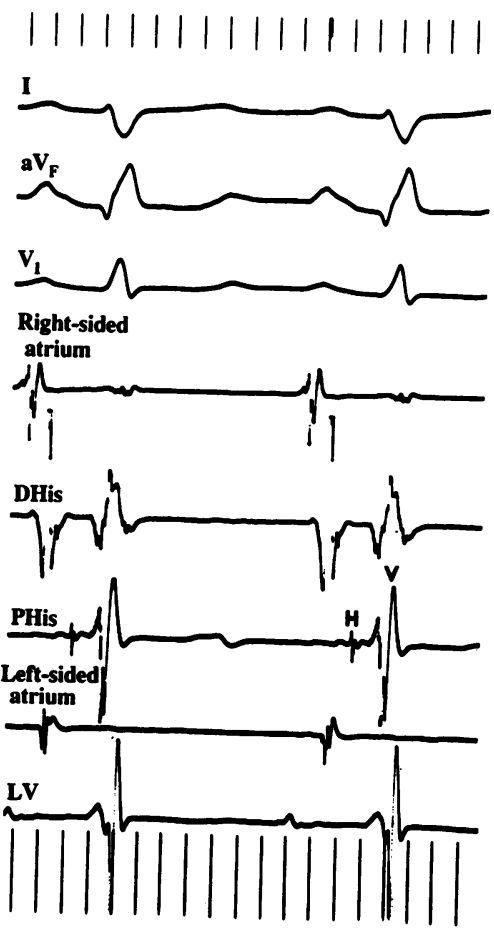

\section{CASE 2}

A 4 year old cyanotic boy had had tachycardia for 3 years. Echocardiography and cardiac catheterisation showed right atrial isomerism, total anomalous pulmonary venous return to a right-sided atrium without obstruction, complete atrioventricular canal, double outlet right ventricle and pulmonary stenosis. An electrocardiogram recorded during an episode of tachycardia showed narrow QRS tachycardia (long PR, short RP) which could be cardioverted by ice bag immersion (fig 3). Alternating forms of the $P$ wave were seen on resting electrocardiograms, and the QRS axis was not changed either during the normal sinus rhythm or rapid atrial pacing. During the electrophysiological study constant QRS configuration during rapid atrial pacing and a continuous $\mathrm{A}_{2} \mathrm{H}_{2} v \mathrm{~A}_{1} \mathrm{~A}_{2}$ curve suggested that anterograde $\mathrm{AV}$ conduction occurred through the posterior AV node only and not the anterior AV node. His potential was recorded near the posterior AV node (fig 2B). After the earliest atrial depolarisation was mapped during tachycardia and ventricular pacing (by electrophysiological study and intracardiac mapping), the clinical tachycardia was attributed to a reentrant circuit conducting anterogradely through the posterior AV node and retrogradely through the anterior $A V$ node. Ventricular pacing and retrograde ventriculoatrial conduction during tachycardia constantly depolarised the atria from the left anteroseptal area (anterior AV node horizon). Therefore, we concluded that the anterior AV node had the property of unidirectional retrograde ventriculoatrial conduction whereas the posterior AV node could conduct only unidirectionally, from the atria to the ventricles. Subsequently, the anterior AV node was surgically ablated during the total cavapulmonary anastomosis operation. The patient died of sepsis 2 weeks after the operation. pathway in a left posterior site (fig $1 \mathrm{~B}$ ). When the paced cycle length was reduced to $340 \mathrm{~ms}$, the AV conduction shifted to the anterior AV node, as suggested by a QS pattern in V1 and normal PR (AV) interval (fig 1C). The Wenckebach cycle length of the anterior AV node was $290 \mathrm{~ms}$. Right ventricular pacing produced only ventriculoatrial dissociation even after an intravenous infusion of isoprenaline had been given to raise the heart rate to $170 / \mathrm{min}$. Atrial and ventricular extrastimuli did not induce any atrioventricular reentrant tachycardia. Short-run atrial flutter-fibrillation only was induced by using $S_{1} S_{1} / S_{1} S_{2}$ at $500 / 210$ ms: the shortest RR interval was 440 $\mathrm{ms}$. The effective refractory period of the anterior AV node was limited by an atrial refractory period of $200 \mathrm{~ms}$. His potential during normal sinus rhythm was recorded near the posterior AV node (fig 2A). Because no tachycardia was induced and the risk of the accessory pathway was deemed to be low, no

\section{Discussion}

Dickinson et al histologically studied the distribution of the conduction system in four patients with right atrial isomerism. There were dual AV nodes. ${ }^{2}$ The posterior AV node was constant in position, lying close to the tendon of Todaro. In patients with two ventricles, the second AV node was anterior in position. A sling of specialised conducting tissue was found at the inferior rim of the ventricular septal defect and in most of the cases made contact with both AV nodes. Ho et al reported similar findings. ${ }^{3}$ However, the electrophysiological properties are still far from clear. The presence of a sling of specialised conducting tissue between the dual AV nodes clearly provides a possible reentrant circuit. We found unidirectional conduction (atrioventricular or ventriculoatrial) in both these dual AV nodes. 

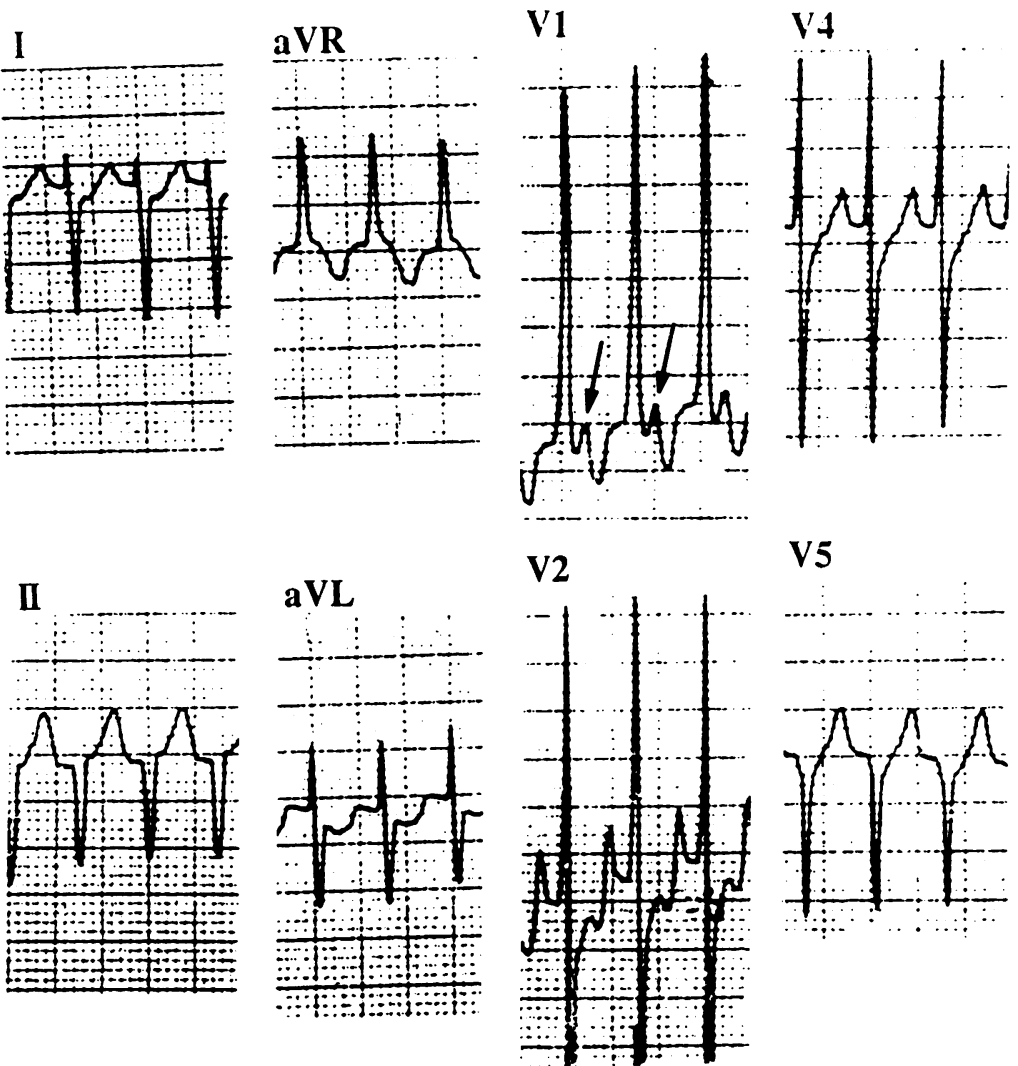

\section{V5}
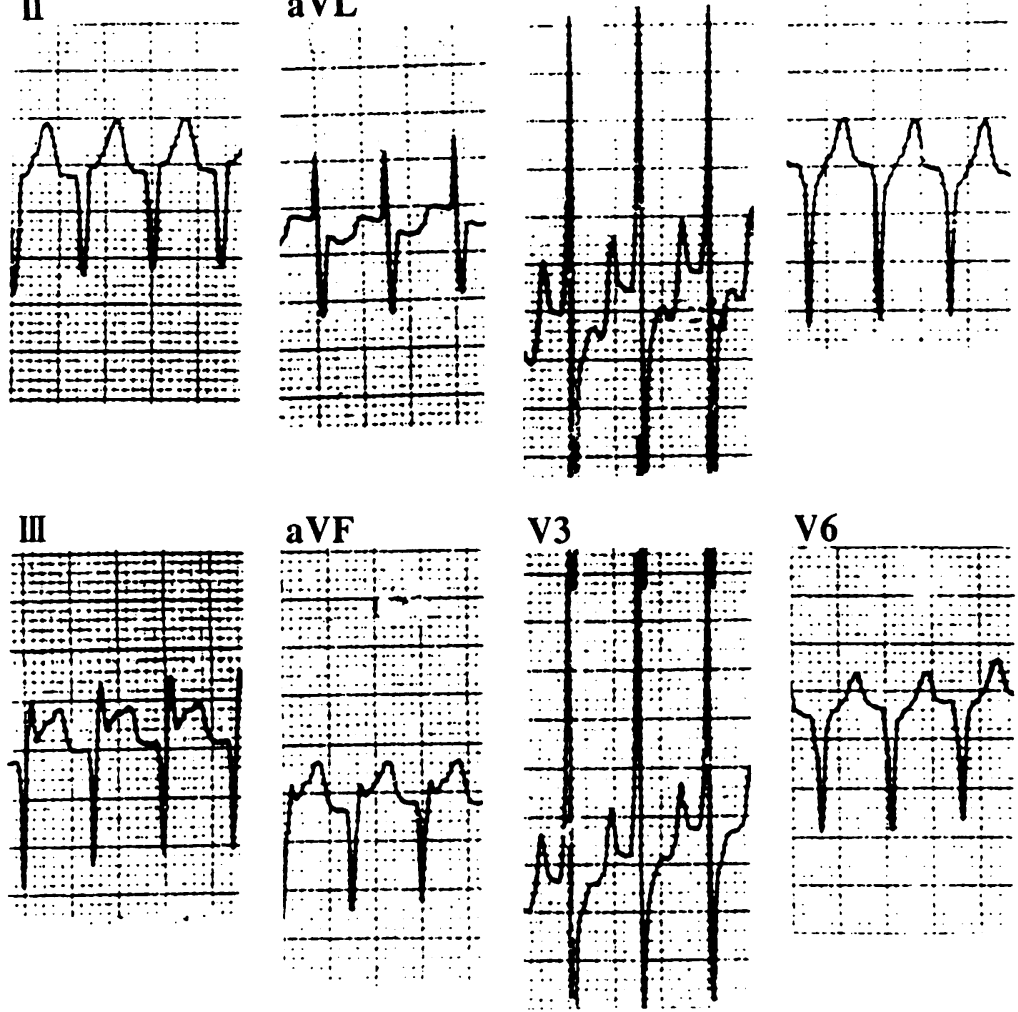

Figure 3 Narrow $Q R S$ tachcardia at a cycle of $280 \mathrm{~ms}$ with a long $P R$ and short $R P$. Arrows indicate retrograde $P$ wave.
The occurrence of AV reentrant tachycardia depends on the direction of unidirectional conduction in the anterior AV node. AV reentrant tachycardia is not common in patients with right atrial isomerism. ${ }^{4}$ Therefore, we speculate that unidirectional conduction may be a common property of the dual AV nodes in right atrial isomerism and that an absence of retrograde ventriculoatrial conduction protects the patients against tachycardia.

The conduction intervals and the refractory periods of the dual AV nodes were all normal for the age of the patient, nonetheless the properties of the anterior and posterior AV nodes were different. Such differences could be the result of different intrinsic properties or different innervation states in the two nodes. Autonomic blockade by atropine and propranolol may help to make the distinction. ${ }^{5}$ However, the possibility that pulmonary flow may increase after the relief of infundibular stenosis by propranolol and may unmask pulmonary venous obstruction should be considered. ${ }^{6}$

1 Van Praagh SV, Santini F, Sanders SP. Cardiac malpositions with special emphasis on visceral heterotax (asplenia and polysplenia syndromes). In: Fyler DC, ed. Nadas' pediatric cardiology. Philadelphia: Hanley \& Belfus, 1992;589-608.

2 Dickinson DF, Wilkinson JL, Anderson KR, Smith A, Ho SY, Anderson RH. The cardiac conduction system in situs ambiguus. Circulation 1979;5:879-85.

3 Ho SY, Fagg N, Anderson RH, Cook A, Allan L Disposition of the atrioventricular conduction tissues in the heart with isomerism of the atrial appendages: its the heart with isomerism of the atrial appendages: its

4 Rudomirsky A, Garson Jr. A. Supraventricular tachycardia. In: Gillette PC, Garson Jr. A, eds. Pediatric arrhythmias: electrophysiology and pacing. Philadelphia: WB Sanders 1990;380-426.

5 Prystowsky EN, Jackman WM, Rinkenberger RL, Heger JJ, Zipes DP. Effect of autonomic blockade on ventricular refractoriness and atrioventricular nodal conduction in humans: evidence supporting a direct cholinergic action humans: evidence supporting a direct cholinergic action on ven

6 Gersony WM. Obstruction to pulmonary venous return obscured by decreased pulmonary blood flow. Chest 1973;64:283-7. 